\title{
Influences on pre-hospital delay in the diagnosis of colorectal cancer: a systematic review
}

\author{
E Mitchell ${ }^{*, 1}$, S Macdonald ${ }^{2}$, NC Campbell ${ }^{3}$, D Weller ${ }^{4}$ and U Macleod ${ }^{2}$ \\ 'School of Health and Social Care, Glasgow Caledonian University, Cowcaddens Road, Glasgow G4 OBA, UK; '2Division of Community Based Sciences, \\ General Practice and Primary Care, University of Glasgow, I Horselethill Road, Glasgow GI 2 9LX, UK; ${ }^{3}$ General Practice and Primary Care, University of \\ Aberdeen, Foresterhill Health Centre, Westburn Road, Aberdeen AB25 2AY, UK; ${ }^{4}$ Community Health Sciences (General Practice), University of Edinburgh, \\ 20 West Richmond Street, Edinburgh EH8 9DX, UK
}

Colorectal cancer is a major global health problem, with survival varying according to stage at diagnosis. Delayed diagnosis can result from patient, practitioner or hospital delay. This paper reports the results of a review of the factors influencing pre-hospital delay - the time between a patient first noticing a cancer symptom and presenting to primary care or between first presentation and referral to secondary care. A systematic methodology was applied, including extensive searches of the literature published from 1970 to 2003, systematic data extraction, quality assessment and narrative data synthesis. Fifty-four studies were included. Patients' non-recognition of symptom seriousness increased delay, as did symptom denial. Patient delay was greater for rectal than colon cancers and the presence of more serious symptoms, such as pain, reduced delay. There appears to be no relationship between delay and patients' age, sex or socioeconomic status. Initial misdiagnosis, inadequate examination and inaccurate investigations increased practitioner delay. Use of referral guidelines may reduce delay, although evidence is currently limited. No intervention studies were identified. If delayed diagnosis is to be reduced, there must be increased recognition of the significance of symptoms among patients, and development and evaluation of interventions that are designed to ensure appropriate diagnosis and examination by practitioners. British Journal of Cancer (2008) 98, 60-70. doi:10.1038/sj.bjc.6604096 www.bjcancer.com

Published online 4 December 2007

(c) 2008 Cancer Research UK

Keywords: colorectal; delay; diagnosis; systematic review

Colorectal cancer is a major global health problem and the fourth most common cause of cancer death worldwide (Parkin et al, 2001). It is also a cancer the incidence of which continues to grow, particularly in developed countries (Parkin et al, 1999). Survival varies according to stage at diagnosis with 5 -year survival falling from almost $90 \%$ for early cancers (Dukes A) to $15 \%$ for advanced tumours, when only palliative resection is possible (McArdle and Hole, 2002). In the majority of cases, primary care is the first point of contact for patients with lower gastrointestinal cancer symptoms, and colorectal cancer is not always an immediate diagnosis. Associated symptoms, including rectal bleeding and altered bowel habit, are common in primary care practice and as such, general practitioners (GPs) are required to differentiate between patients whose symptoms may be due to cancer and the much larger number of patients whose symptoms are attributable to benign, self-limiting illness.

The complexity surrounding identification of those patients requiring further investigation has led to the production of guidelines in many countries, all with the aim of encouraging earlier diagnosis (Benson et al, 2000; Department of Health, 2000; National Institute for Health and Clinical Excellence, 2005; Australian Cancer Network, 2006). In addition, patients too must

*Correspondence: Dr E Mitchell; E-mail: liz.mitchell2@googlemail.com Received 19 July 2007; revised 18 October 2007; accepted 22 October 2007; published online 4 December 2007 determine whether a symptom warrants presentation to the health service or requires adoption of a 'wait and see approach'. As such, delayed diagnosis of colorectal cancer can occur as a result of patient delay (the time between first noticing a symptom and first consulting a doctor), practitioner delay (between first consultation and referral) or hospital delay (between referral and diagnosis) (Nichols et al, 1981) and greater knowledge of the factors contributing to these phases is required if survival is to be increased. We conducted a systematic review of the factors that influence pre-hospital delay, that is, up to the point of referral.

\section{MATERIALS AND METHODS}

A worldwide review of the literature from 1970 to 2003 was conducted to identify the reasons for delay by patients in presentation with cancer symptoms and by practitioners in cancer detection and referral. Studies were identified from electronic databases (Medline, EMBASE, CINAHL, PsycINFO, Science Citation Index, Social Science Citation Index, International Bibliography of the Social Sciences, Proceedings First and Web of Science Proceedings), Cochrane Collaboration review groups, bibliographies, books, citations in identified articles and authors active in the field. Studies were selected if they focused on adult cancer and (1) the participants were individuals or groups of patients or primary care practitioners and (2) they evaluated factors associated with the interval between a patient first noticing 
a cancer symptom and presenting to primary care, or described an intervention designed to reduce that interval or (3) they evaluated factors associated with the time interval between the patient first presenting to primary care and being referred to secondary care, or described an intervention designed to reduce that interval. To identify factors associated with help-seeking and referral behaviour, studies that determined patient attitudes towards cancer awareness and presentation were also included, as were those which determined provider attitudes or behaviour towards cancer referral. Studies evaluating delay from presentation to treatment were not excluded until they were reviewed to ensure that they did not differentiate between stages in the delay cycle. Studies assessing the outcome of delay in terms of diagnosis, treatment or patient outcomes were excluded, as were those considering only the cost of interventions, validity of referral decisions or differences in referral periods.

Following the initial search, all references were independently assessed, and if subsequently eligible for inclusion, rated by two reviewers (SM, UM). Where differences of opinion occurred, papers were validated by a third reviewer (EM) and findings discussed until a consensus was reached. Previously developed scoring systems were extended and applied to assess the methodological adequacy of studies (Mitchell and Sullivan, 2001). Many used methodologies that did not lend themselves to such techniques; therefore, each included study was also assessed on the strength of the evidence it presented. We determined three grades of evidence: strong, moderate and insufficient.

Studies providing strong evidence were those with an adequate sample size, rigorous methods to ascertain data (i.e. not open to selection bias) and reporting statistically significant differences in relation to the delay-related factors identified (or using appropriate analytic techniques if qualitative). Studies providing moderate evidence had an adequate sample size, reported significant differences but used less rigorous methods to ascertain data or had an adequate sample size, used rigorous methods to ascertain data but used comparative analysis or reported only relevant descriptive statistics, without statistical testing of differences. Studies providing insufficient evidence had unclear or inappropriate methods to ascertain data and insufficient analysis. Where a study inferred results, the strength of its evidence was downgraded. The full methods used in this review have been described in detail elsewhere (Macdonald et al, 2006). Narrative synthesis of findings was undertaken to identify key concepts and themes that were shared across individual studies.

\section{RESULTS}

The search strategy identified 28356 articles of which only 169 $(0.6 \%)$ met the inclusion criteria and were subject to detailed review (Figure 1). Fifty-four papers were included in the final analysis. Cohen's kappa was used to determine inter-rater reliability, that is, the level of concurrence between the two independent reviewers in relation to whether identified studies were considered eligible for detailed evaluation. Kappa co-efficient for agreement beyond chance was 0.52 .

More than half of the included studies $(n=31)$ were carried out in Western Europe, over half of these in the UK. None employed a controlled trial methodology, with most involving review of medical records or structured interviews with patients. More than one-third investigated both patient and practitioner delay $(n=20)$, almost half studied patient factors only $(n=25)$ and the remainder studied practitioner factors only. Studies most commonly evaluated any colorectal cancer, with smaller numbers dealing specifically with cancer of the rectum $(11 \%)$, colon $(9 \%)$ or anus (4\%). Twenty-six papers were assessed as providing strong evidence, 19 provided moderate evidence and 9 provided insufficient evidence.

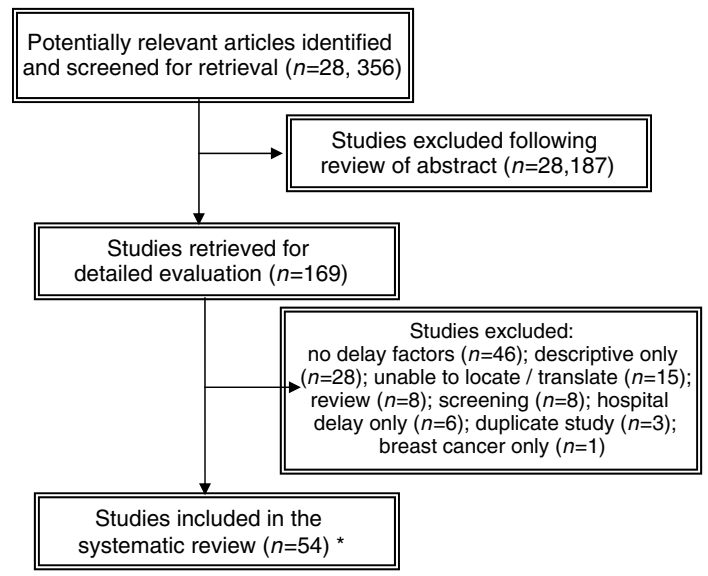

${ }^{*} n=54$ (12 papers related to more than one cancer group)

Figure I Flow of studies into the review.

Studies were comparatively small in size, involving between 17 and 2525 participants (mean 420; median 228). The period under study ranged from 3 months to 53 years. Although more than half of the studies considered practitioner-related delay factors, only five included primary care practitioners as subjects. In almost three-quarters of studies, participants were identified from secondary care $(n=38)$, and were either in-patients $(39 \%)$, outpatient attendees (16\%), a combination of the two $(5 \%)$ or identified from hospital records (37\%). Other sources used were cancer registries, census or other household directories and patient groups. Only 6 of the 54 studies recruited patients from primary care.

\section{Delay intervals}

Thirty-eight studies reported length of delay, either from patient recognition of symptoms to presentation $(n=36)$ or from presentation to practitioner referral $(n=24)$. This was reported in a non-standardised way, and less than half reported intervals in medians, despite delay times having typically skewed distributions. The most frequently used methods of deriving delay intervals were by structured patient interview or data abstraction from hospital records. Only five studies used primary care records (9\%). Median patient delay ranged from 7 days to 5 months (Worden and Weisman, 1975; Turunen and Peltokallio, 1982; MacArthur and Smith, 1984; Funch, 1985; Robinson et al, 1986; Ratcliffe et al, 1989; Dent et al, 1990; Curless et al, 1994; Arbman et al, 1996; Porta et al, 1996; Mulcahy and O’Donoghue, 1997; Majumdar et al, 1999; Mariscal et al, 2001) and practitioner delay from 0 to 15 months (Turunen and Peltokallio, 1982; MacArthur and Smith, 1984; Funch, 1985; Ratcliffe et al, 1989; Mansson, 1990; Jones and Dudgeon, 1992; Curless et al, 1994; Arbman et al, 1996; Harris and Simson, 1998; Majumdar et al, 1999; Mariscal et al, 2001).

\section{Factors influencing patient delay}

Forty-four papers considered factors that influenced patient delay. Most $(n=41)$ identified factors that increased delay, whereas almost two-thirds $(n=27)$ identified factors that decreased delay (Figure 2).

\section{Patient behaviour}

The influence of symptom awareness, and more particularly patients' interpretation of symptoms, was a common theme across studies. Non-recognition of the seriousness of symptoms (Worden 


\begin{tabular}{lr}
\hline Decreases & Nil effect \\
\hline Symptoms & Increases \\
\hline *Pain & *Altered bowel habit \\
& $*$ Site (rectum) \\
\hline Patient history & \\
\hline$* *$ Comorbidity & \\
\hline
\end{tabular}

\begin{tabular}{|c|c|c|}
\hline \multicolumn{3}{|c|}{ Patient characteristics } \\
\hline & *Age (older) & *Education level (lower) \\
\hline & *Sex (male) & **Rural residence \\
\hline *Social support & $\begin{array}{c}\text { *Socioeconomic } \\
\text { status (lower) }\end{array}$ & \\
\hline
\end{tabular}

Patient behaviour

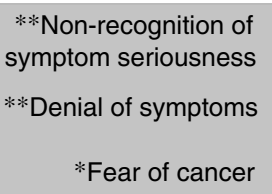

Factor included only if supported by studies providing strong or moderate levels of evidence

*Found in $\geqslant 50 \%$ of studies considering factor; **found in $\geqslant 75 \%$ of studies considering factor

Figure 2 Main factors associated with patient delay and direction of influence.

and Weisman, 1975; Holliday and Hardcastle, 1979; Rubin et al, 1980; Anon, 1982; Marshall and Funch, 1986; Mor et al, 1990; Prohaska et al, 1990; Byles et al, 1992; Curless et al, 1994; Porta et al, 1996; Roncoroni et al, 1999; Sladden et al, 1999; Young et al, 2000; de Nooijer et al, 2001; Cockburn et al, 2003), or lack of knowledge, either about the disease itself or about the availability of screening was a major contributor to increased delay (Anon, 1982, 1986; Camilleri-Brennan and Steele, 1999; Pullyblank et al, 2002; McCaffery et al, 2003). Patients who presented late tended to either deny their symptoms entirely, or redefine these in relation to benign disease (Worden and Weisman, 1975; Bain et al, 2002; Langenbach et al, 2003). Perhaps unsurprisingly, increased delay was also found for patients who were less worried about their symptoms (Dent et al, 1990) or who self-diagnosed or selfmedicated before presenting to primary care (Funch, 1985; Dent et al, 1990; Tanum et al, 1991; Byles et al, 1992; Sladden et al, 1999; Cockburn et al, 2003) (Table 1).

The anxiety associated with recognising a potential cancer symptom was also a key factor in the decision to present. Fear that symptoms were indicative of cancer (Prohaska et al, 1990; Byles et al, 1992; de Nooijer et al, 2001), fear of investigations related to diagnosis of cancer (Langenbach et al, 2003) and fear of powerlessness (Worden and Weisman, 1975; McCaffery et al, 2003) made patients consult less quickly, although, for some, fear that a symptom might be a sign of cancer brought about more rapid presentation (Hackett et al, 1973; Dent et al, 1990; Sladden et al, 1999; de Nooijer et al, 2001).

\section{Presenting symptom and patient history}

For the most part, patients who suffered from more serious symptoms such as obstruction or abdominal pain delayed less (Devlin et al, 1973; MacAdam, 1979; Rubin et al, 1980; MacArthur and Smith, 1984; Prohaska et al, 1990; Mulcahy and O'Donoghue, 1997; Majumdar et al, 1999; Young et al, 2000; Mariscal et al, 2001), whereas those experiencing either nonspecific symptoms or more common symptoms, such as bleeding or altered bowel habit, delayed longer (Devlin et al, 1973; Galloway et al, 1984; Mor et al, 1990; Curless et al, 1994). There were, however, some patients for whom pain resulted in increased delay (Hackett et al, 1973; Nilsson et al, 1982; MacArthur and Smith, 1984; Prohaska et al, 1990). Those who recognised the symptom or who had previous experience of a symptom or of cancer itself tended to delay less (MacDonald and Freeling, 1986; Samet et al, 1988; Dent et al, 1990; Porta et al, 1996; Sladden et al, 1999). This was also the case for those with comorbidity (Ratcliffe et al, 1989; Mor et al, 1990; Porta et al, 1996; Mariscal et al, 2001) and those experiencing multiple symptoms (Mariscal et al, 2001).

A number of studies considered the relationship between presentation behaviour and cancer site. These demonstrated that those with cancer of the rectum were more likely to have delayed than patients with colon cancer (Hackett et al, 1973; Ratcliffe et al, 1989; Arbman et al, 1996; Mulcahy and O’Donoghue, 1997; Harris and Simson, 1998). In addition, there was some evidence to suggest that patients with colon cancer may delay less than patients with other cancers such as melanoma (de Nooijer et al, 2001).

\section{Patient characteristics}

Social networks and support were identified as being a potentially important factor in reducing delay, when patients either sought advice from or made decisions based on the experience of others (Holliday and Hardcastle, 1979; MacAdam, 1979; MacArthur and Smith, 1984; Samet et al, 1988; Camilleri-Brennan and Steele, 1999; Roncoroni et al, 1999; Sladden et al, 1999). By and large, patient age (Worden and Weisman, 1975; MacAdam, 1979; McDermott et al, 1981; Turunen and Peltokallio, 1982; Pitluk and Poticha, 1983; Galloway et al, 1984; MacArthur and Smith, 1984; Marshall and Funch, 1986; Robinson et al, 1986; Samet et al, 1988; Dent et al, 1990; Mor et al, 1990; Prohaska et al, 1990; Marble et al, 1992; Kemppainen et al, 1993; Curless et al, 1994; Arbman et al, 1996; Porta et al, 1996; Mulcahy and O'Donoghue, 1997; Majumdar et al, 
Table I Patient associated delay factors

\begin{tabular}{|c|c|c|c|c|c|c|c|c|}
\hline Author(s) & Location & Study type & Participants & Cancer site & Factors which increase delay & Factors which decrease delay & No impact on delay & Evidence \\
\hline $\begin{array}{l}\text { Devlin et al } \\
\text { (1973) }\end{array}$ & England & $\begin{array}{l}\text { Retrospective } \\
\text { observational }\end{array}$ & $\begin{array}{l}310 \text { patients (aged } 30-95,53 \% \\
\text { men, } 47 \% \text { women) }\end{array}$ & Rectum & $\begin{array}{l}\text { Symptom type - altered bowel } \\
\text { habit, bleeding }\end{array}$ & $\begin{array}{l}\text { Symptom type - abdominal or ano- } \\
\text { rectal pain }\end{array}$ & & Moderate \\
\hline $\begin{array}{l}\text { Hackett et al } \\
\text { (1973) }\end{array}$ & $\begin{array}{l}\text { Massachusetts, } \\
\text { USA }\end{array}$ & $\begin{array}{l}\text { Prospective } \\
\text { observational }\end{array}$ & $\begin{array}{l}563 \text { patients (aged } 17-91 \text {, mean } \\
62 ; 46 \% \text { men, } 54 \% \text { women); } 17 \% \\
\text { with colon/rectal cancer) }\end{array}$ & Colon, rectum & $\begin{array}{l}\text { Symptom type - pain; cancer } \\
\text { site - rectum; social class - lower; } \\
\text { procrastination; worry over health; } \\
\text { family history }\end{array}$ & $\begin{array}{l}\text { Worry; incapacitated by symptoms; } \\
\text { acknowledgment of cancer }\end{array}$ & & Strong \\
\hline $\begin{array}{l}\text { Worden and } \\
\text { Weisman } \\
\text { (1975) }\end{array}$ & $\begin{array}{l}\text { Massachusetts, } \\
\text { USA }\end{array}$ & $\begin{array}{l}\text { Prospective } \\
\text { observational }\end{array}$ & $\begin{array}{l}\text { I } 25 \text { patients (aged } 19-59,38 \% \\
\text { men, } 62 \% \text { women), } 22 \% \text { with colon } \\
\text { cancer }\end{array}$ & Colon & $\begin{array}{l}\text { Non-recognition of symptom } \\
\text { seriousness; denial; powerlessness; } \\
\text { comorbidity; fatigue }\end{array}$ & & $\begin{array}{l}\text { Age; sex; marital status; } \\
\text { socioeconomic status; family history }\end{array}$ & Strong \\
\hline $\begin{array}{l}\text { Holliday and } \\
\text { Hardcastle } \\
\text { (1979) }\end{array}$ & England & $\begin{array}{l}\text { Prospective } \\
\text { observational }\end{array}$ & $\begin{array}{l}200 \text { patients ( } 58 \% \text { men, mean age } \\
66 ; 42 \% \text { women mean age } 67)\end{array}$ & Colorectal & $\begin{array}{l}\text { Non-recognition of symptom } \\
\text { seriousness }\end{array}$ & $\begin{array}{l}\text { Advice from social } \\
\text { network }\end{array}$ & & Moderate \\
\hline $\begin{array}{l}\text { MacAdam } \\
\text { (1979) }\end{array}$ & England & $\begin{array}{l}\text { Prospective } \\
\text { observational }\end{array}$ & $\begin{array}{l}\text { I } 50 \text { patients (79\% with } \\
\text { colon/rectal cancer), I05 GPs }\end{array}$ & Colon, rectum & Cancer site - rectum & $\begin{array}{l}\text { Cancer site - colon; symptom type } \\
\text { - abdominal pain, bleeding }\end{array}$ & $\begin{array}{l}\text { Socioeconomic status; } \\
\text { age; sex; social isolation; frequency } \\
\text { of consulting }\end{array}$ & Moderate \\
\hline $\begin{array}{l}\text { Rubin et al } \\
\text { (1980) }\end{array}$ & Israel & $\begin{array}{l}\text { Prospective } \\
\text { observational }\end{array}$ & $\begin{array}{l}100 \text { patients (aged } 36-85, \text { mean } \\
64 ; 66 \% \text { men, } 34 \% \text { women) }\end{array}$ & Colorectal & $\begin{array}{l}\text { Non-recognition of symptom } \\
\text { seriousness }\end{array}$ & $\begin{array}{l}\text { Symptom type - abdominal pain, } \\
\text { weakness }\end{array}$ & & Strong \\
\hline $\begin{array}{l}\text { McDermott } \\
\text { et al }(1981)\end{array}$ & Australia & $\begin{array}{l}\text { Retrospective } \\
\text { observational }\end{array}$ & $\begin{array}{l}1228 \text { patients ( } 55 \% \text { men, mean age } \\
61 ; 45 \% \text { women, mean age } 59 \text { ) }\end{array}$ & Rectum & & & Age; sex; symptom type & Moderate \\
\hline Anon (1982) & USA & Cross-sectional & $\begin{array}{l}804 \text { members of the public (aged } \\
40+)\end{array}$ & Colorectal & $\begin{array}{l}\text { Non-recognition of symptom } \\
\text { seriousness; lack of knowledge; } \\
\text { lack of routine screening }\end{array}$ & & & Insufficient \\
\hline $\begin{array}{l}\text { Nilsson et al } \\
\text { (1982) }\end{array}$ & Sweden & $\begin{array}{l}\text { Retrospective } \\
\text { observational }\end{array}$ & $\begin{array}{l}284 \text { patients (aged } 20-99 ; 52 \% \\
\text { men, } 48 \% \text { women) }\end{array}$ & Colorectal & $\begin{array}{l}\text { Symptom type - pain, bleeding, } \\
\text { bowel disturbance }{ }^{\mathrm{a}}\end{array}$ & & & Insufficient \\
\hline $\begin{array}{l}\text { Turunen and } \\
\text { Peltokallio } \\
\text { (1982) }\end{array}$ & Finland & $\begin{array}{l}\text { Prospective } \\
\text { observational }\end{array}$ & $\begin{array}{l}100 \text { patients ( } 45 \% \text { men, } 55 \% \\
\text { women }\end{array}$ & Colorectal & Age $-<50 ;$ sex - male & & & Moderate \\
\hline $\begin{array}{l}\text { Pitluk and } \\
\text { Poticha (1983) }\end{array}$ & Illinois, USA & $\begin{array}{l}\text { Retrospective } \\
\text { observational }\end{array}$ & $\begin{array}{l}826 \text { patients ( } 31 \text { aged } \leqslant 40 ; 45 \% \\
\text { men, } 55 \% \text { women) }\end{array}$ & Colorectal & Age - younger & & & Moderate \\
\hline $\begin{array}{l}\text { Galloway et al } \\
\text { (1984) }\end{array}$ & Scotland & $\begin{array}{l}\text { Retrospective } \\
\text { observational }\end{array}$ & $\begin{array}{l}48 \text { I patients ( } 92.5 \% \text { aged } 50+; 50 \% \\
\text { men, } 50 \% \text { women) }\end{array}$ & \% Colorectal & $\begin{array}{l}\text { Age - younger; symptom } \\
\text { type - rectal bleeding }\end{array}$ & & & Strong \\
\hline $\begin{array}{l}\text { MacArthur and } \\
\text { Smith (1984) }\end{array}$ & England & $\begin{array}{l}\text { Prospective } \\
\text { observational }\end{array}$ & 127 patients & Colorectal & $\begin{array}{l}\text { Symptom type - weight loss, } \\
\text { rectal pain; }\end{array}$ & $\begin{array}{l}\text { Symptom type - abdominal pain, } \\
\text { nausea; advice from social network }\end{array}$ & Age; social class & Strong \\
\hline Funch (1985) & $\begin{array}{l}\text { Washington, } \\
\text { USA }\end{array}$ & $\begin{array}{l}\text { Prospective } \\
\text { observational }\end{array}$ & $\begin{array}{l}294 \text { patients (aged I } 8-85 ; 49 \% \\
\text { men, } 51 \% \text { women) }\end{array}$ & Colorectal & $\begin{array}{l}\text { Other life events; feeling better; } \\
\text { self-treatment }\end{array}$ & & & Moderate \\
\hline Anon (1986) & USA & Cross-sectional & $\begin{array}{l}2525 \text { members of the public (aged } \\
40+\text { ) }\end{array}$ & Colorectal & $\begin{array}{l}\text { Lack of awareness of screening; } \\
\text { lack of knowledge }\end{array}$ & & & Insufficient \\
\hline $\begin{array}{l}\text { MacDonald and } \\
\text { Freeling (1986) }\end{array}$ & d England & Cross-sectional & $\begin{array}{l}\text { 17I GP patients (aged 55+; 50\% } \\
\text { men, } 50 \% \text { women) }\end{array}$ & Colorectal & & $\begin{array}{l}\text { Recognition of symptoms; } \\
\text { symptom type - bleeding }\end{array}$ & & Moderate \\
\hline $\begin{array}{l}\text { Marshall and } \\
\text { Funch (1986) }\end{array}$ & $\begin{array}{l}\text { Washington, } \\
\text { USA }\end{array}$ & $\begin{array}{l}\text { Prospective } \\
\text { observational }\end{array}$ & $\begin{array}{l}306 \text { patients (aged 18-85; 50\% } \\
\text { men, } 50 \% \text { women) }\end{array}$ & Colorectal & $\begin{array}{l}\text { Sex - female; cancer } \\
\text { site - rectum (female)/colon } \\
\text { (male); non-recognition of } \\
\text { symptom seriousness }\end{array}$ & & Age; education & Strong \\
\hline $\begin{array}{l}\text { Robinson et al } \\
\text { (1986) }\end{array}$ & Israel & $\begin{array}{l}\text { Retrospective } \\
\text { observational }\end{array}$ & $\begin{array}{l}445 \text { patients ( } 54 \% \text { men, } 46 \% \\
\text { women) }\end{array}$ & Colorectal & Widowhood & Residence - urban & Age; sex & Strong \\
\hline $\begin{array}{l}\text { Samet et al } \\
\text { (1988) }\end{array}$ & $\begin{array}{l}\text { New Mexico, } \\
\text { USA }\end{array}$ & $\begin{array}{l}\text { Prospective } \\
\text { observational }\end{array}$ & $\begin{array}{l}800 \text { patients (aged } 65-100 \text {, mean } \\
72 \text { ), } 28 \% \text { with colorectal cancer }\end{array}$ & Colorectal & $\begin{array}{l}\text { Race - white Hispanic; } \\
\text { sex - male; income - lower }\end{array}$ & $\begin{array}{l}\text { Previous cancer diagnosis; regular } \\
\text { check-ups }\end{array}$ & $\begin{array}{l}\text { Age; availability of vehicle; social } \\
\text { support; participation in screening }\end{array}$ & Strong \\
\hline $\begin{array}{l}\text { Ratcliffe } \\
\text { et al (1989) }\end{array}$ & England & $\begin{array}{l}\text { Prospective } \\
\text { observational }\end{array}$ & $\begin{array}{l}332 \text { patients (aged } 30-100 \text {, mean } \\
70 ; 51 \% \text { men, } 49 \% \text { women) }\end{array}$ & Colorectal & $\begin{array}{l}\text { Family history; cancer } \\
\text { site - rectum }\end{array}$ & Comorbidity - diverticular disease & & Strong \\
\hline $\begin{array}{l}\text { Dent et al } \\
\text { (1990) }\end{array}$ & Australia & Cross-sectional & $\begin{array}{l}93 \text { patients with rectal bleeding } \\
\text { (aged } 35-85 \text {, median } 55 ; 54 \% \\
\text { men, } 46 \% \text { women), } 58 \text { GPs }\end{array}$ & Colorectal & $\begin{array}{l}\text { Consulting non-medical } \\
\text { professional; self-treatment; } \\
\text { less worry (self-diagnosis); } \\
\text { education level - lower }\end{array}$ & $\begin{array}{l}\text { Previous rectal bleeding; regularly } \\
\text { checking toilet paper or faeces; } \\
\text { worry } \\
\text { that bleeding means cancer }\end{array}$ & $\begin{array}{l}\text { Age; sex; social support; income; } \\
\text { ethnicity; occupation }\end{array}$ & Strong \\
\hline
\end{tabular}

\section{Clinical Studies}


Table I (Continued)

\begin{tabular}{|c|c|c|c|c|c|c|c|c|}
\hline Author(s) & Location & Study type & Participants & Cancer site & Factors which increase delay & Factors which decrease delay & No impact on delay & Evidence \\
\hline $\begin{array}{l}\text { Mor et al } \\
(1990)\end{array}$ & $\begin{array}{l}\text { Rhode Island, } \\
\text { USA }\end{array}$ & $\begin{array}{l}\text { Prospective } \\
\text { observational }\end{array}$ & $\begin{array}{l}625 \text { patients (aged } 45-90 ; \\
31 \% \text { men, } 69 \% \text { women), } \\
46 \% \text { with colorectal cancer }\end{array}$ & Colorectal & $\begin{array}{l}\text { Non-recognition of symptom } \\
\text { seriousness; age - younger; } \\
\text { symptom type - bleeding, altered } \\
\text { bowel habit }\end{array}$ & Comorbidity & & Strong \\
\hline $\begin{array}{l}\text { Prohaska } \\
\text { et al (1990) }\end{array}$ & $\begin{array}{l}\text { Washington, } \\
\text { USA }\end{array}$ & $\begin{array}{l}\text { Prospective } \\
\text { observational }\end{array}$ & $\begin{array}{l}254 \text { patients ( } 48 \% \text { men; } \\
52 \% \text { women) }\end{array}$ & Colon, rectum & $\begin{array}{l}\text { Non-recognition of symptom } \\
\text { seriousness; symptom } \\
\text { type - rectal pain; too busy; } \\
\text { fear }\end{array}$ & Symptom type - abdominal pain & Age; income & Strong \\
\hline $\begin{array}{l}\text { Tanum et al } \\
(|99|)\end{array}$ & Norway & $\begin{array}{l}\text { Retrospective } \\
\text { observational }\end{array}$ & $\begin{array}{l}117 \text { patients (aged } 35-91 \\
21 \% \text { men, } 79 \% \text { women) }\end{array}$ & Anus & Self-treatment & & & Insufficient \\
\hline $\begin{array}{l}\text { Byles et al } \\
\text { (1992) }\end{array}$ & Australia & Cross-sectional & $\begin{array}{l}1221 \text { members of public (aged } \\
40+; 49 \% \text { men; } 51 \% \text { women), } \\
20 \% \text { with rectal bleeding }\end{array}$ & Rectum & $\begin{array}{l}\text { Non-recognition of symptom } \\
\text { seriousness; embarrassment; fear; } \\
\text { self-diagnosis }\end{array}$ & & & Moderate \\
\hline $\begin{array}{l}\text { Marble et al } \\
\text { (1992) }\end{array}$ & $\begin{array}{l}\text { Connecticut, } \\
\text { USA }\end{array}$ & $\begin{array}{l}\text { Retrospective } \\
\text { observational }\end{array}$ & $\begin{array}{l}\text { I } 00 \text { patients ( } 50 \text { aged } 14-40 \\
\text { mean } 36 ; 50 \text { aged } 49-86 \\
\text { mean } 70 \text { ) }\end{array}$ & Colorectal & Age - younger & & & Strong \\
\hline $\begin{array}{l}\text { Kemppainen } \\
\text { et al (1993) }\end{array}$ & Finland & $\begin{array}{l}\text { Retrospective } \\
\text { observational }\end{array}$ & $\begin{array}{l}\text { I } 78 \text { patients (aged } 27-97 \\
\text { mean } 91 ; 44 \% \text { men, } 56 \% \\
\text { women) }\end{array}$ & Colorectal & $\begin{array}{l}\text { Age and sex }- \text { male }<65 \text {, female } \\
80+\end{array}$ & & & Moderate \\
\hline $\begin{array}{l}\text { Vineis et al } \\
\text { (1993) }\end{array}$ & Italy & $\begin{array}{l}\text { Prospective } \\
\text { observational }\end{array}$ & $\begin{array}{l}330 \text { patients, } 29 \% \text { with colon } \\
\text { cancer ( } 58 \% \text { men, } 42 \% \text { women) }\end{array}$ & Colon & & Education level - higher & & Strong \\
\hline $\begin{array}{l}\text { Curless et al } \\
\text { (1994) }\end{array}$ & England & $\begin{array}{l}\text { Prospective } \\
\text { observational }\end{array}$ & $\begin{array}{l}273 \text { patients (aged } 25-93 \text {, } \\
\text { median } 68 ; 56 \% \text { men, } 44 \% \\
\text { women) }\end{array}$ & Colorectal & $\begin{array}{l}\text { Presentation with non-specific } \\
\text { symptoms; non-recognition } \\
\text { of symptom seriousness }\end{array}$ & & Age & Strong \\
\hline $\begin{array}{l}\text { Arbman et al } \\
\text { (1996) }\end{array}$ & Sweden & $\begin{array}{l}\text { Retrospective } \\
\text { observational }\end{array}$ & $\begin{array}{l}554 \text { patients (aged } 30-95 ; 51 \% \\
\text { men, mean age } 70 ; 49 \% \text { women, } \\
\text { mean age } 72 \text { ), } 39 \% \text { with rectal, } \\
61 \% \text { with colon cancer }\end{array}$ & Colon, rectum & Cancer site - rectum; & Presenting as emergency & Age & Strong \\
\hline $\begin{array}{l}\text { Porta et al } \\
\text { (1996) }\end{array}$ & Spain & $\begin{array}{l}\text { Prospective } \\
\text { observational }\end{array}$ & $\begin{array}{l}183 \text { patients (mean age } 67 \\
66 \% \text { men, } 34 \% \text { women) }\end{array}$ & Colon, rectum & $\begin{array}{l}\text { Age - older; sex - male; illiteracy; } \\
\text { social class - lower; unemployment; } \\
\text { non-recognition of symptom } \\
\text { seriousness }\end{array}$ & $\begin{array}{l}\text { Age - younger, comorbidity; } \\
\text {; recognition } \\
\text { of symptom seriousness }\end{array}$ & $\begin{array}{l}\text { Marital status; family } \\
\text { history }\end{array}$ & Strong \\
\hline $\begin{array}{l}\text { Mulcahy and } \\
\text { O'Donoghue } \\
\text { (1997) }\end{array}$ & Ireland & $\begin{array}{l}\text { Prospective } \\
\text { observational }\end{array}$ & $\begin{array}{l}777 \text { patients (aged } 26-92 \\
\text { mean } 68 ; 54 \% \text { men, } 46 \% \\
\text { women) }\end{array}$ & Colorectal & $\begin{array}{l}\text { Age }- \text { younger; cancer } \\
\text { site - rectum }\end{array}$ & $\begin{array}{l}\text { Symptom } \\
\text { type - obstruction }\end{array}$ & Sex & Strong \\
\hline $\begin{array}{l}\text { Camilleri- } \\
\text { Brennan and } \\
\text { Steele (1999) }\end{array}$ & Scotland & Cross-sectional & $\begin{array}{l}1004 \text { adult members of the } \\
\text { public (mean age 50, } 40 \% \text { men, } \\
60 \% \text { women) }\end{array}$ & Colorectal & Lack of knowledge $\mathrm{e}^{\mathrm{a}}$ & Experience through social network ${ }^{a}$ & & Moderate \\
\hline $\begin{array}{l}\text { Majumdar } \\
\text { et al (1999) }\end{array}$ & $\begin{array}{l}\text { North Carolina, } \\
\text { USA }\end{array}$ & $\begin{array}{l}\text { Retrospective } \\
\text { observational }\end{array}$ & $\begin{array}{l}\text { I } 94 \text { patients (aged } 15-95 \\
\text { mean } 66 ; 53 \% \text { men, } 47 \% \text { women) }\end{array}$ & Colorectal & Symptom type - weight loss & $\begin{array}{l}\text { Symptom } \\
\text { type - obstruction }\end{array}$ & Age; sex; cancer site & Strong \\
\hline $\begin{array}{l}\text { Roncoroni } \\
\text { et al (1999) }\end{array}$ & Italy & $\begin{array}{l}\text { Prospective } \\
\text { observational }\end{array}$ & $\begin{array}{l}100 \text { patients (aged } 38-89 \\
54 \% \text { men, } 46 \% \text { women) }\end{array}$ & Colorectal & $\begin{array}{l}\text { Non-recognition of symptom } \\
\text { seriousness }\end{array}$ & Advice from social network & & Strong \\
\hline $\begin{array}{l}\text { Sladden } \\
\text { et al (1999) }\end{array}$ & Australia & Cross-sectional & $\begin{array}{l}903 \mathrm{GP} \text { attenders (aged 50+, } \\
\text { mean 66; } 44 \% \text { men, } 56 \% \text { women) }\end{array}$ & Rectum & $\begin{array}{l}\text { Non-recognition of symptom } \\
\text { seriousness; self-treatment; }\end{array}$ & $\begin{array}{l}\text { Blood in toilet; advice from social } \\
\text { network; worry that bleeding } \\
\text { means cancer }\end{array}$ & & Strong \\
\hline $\begin{array}{l}\text { Young et al } \\
(2000)\end{array}$ & Australia & $\begin{array}{l}\text { Prospective } \\
\text { observational }\end{array}$ & $\begin{array}{l}\text { I } 00 \text { patients (aged } 43-92 \\
\text { mean } 70 ; 52 \% \text { men, } 48 \% \text { women) }\end{array}$ & Colorectal & $\begin{array}{l}\text { Sex - male; non-recognition of } \\
\text { symptom seriousness }\end{array}$ & Symptom type - pain, bleeding & & Strong \\
\hline $\begin{array}{l}\text { de Nooijer } \\
\text { et al (200I) }\end{array}$ & $\begin{array}{l}\text { The } \\
\text { Netherlands }\end{array}$ & $\begin{array}{l}\text { Qualitative } \\
\text { interviews }\end{array}$ & $\begin{array}{l}23 \text { patients (mean age } 52 ; \\
43 \% \text { men, } 57 \% \text { women), } 26 \% \\
\text { with colon cancer. } 10 \mathrm{GPs}\end{array}$ & Colon & $\begin{array}{l}\text { Non-recognition of symptom } \\
\text { seriousness; cancer-site - colon; } \\
\text { fear of cancer }\end{array}$ & Fear of cancer; trust in GP & & Strong \\
\hline $\begin{array}{l}\text { Mariscal et al } \\
(200 \mathrm{I})\end{array}$ & Spain & $\begin{array}{l}\text { Prospective } \\
\text { observational }\end{array}$ & $\begin{array}{l}217 \text { patients (aged } 59-74, \text { mean } \\
65 ; 59 \% \text { men, } 41 \% \text { women), } 73 \% \\
\text { with colon cancer }\end{array}$ & Large bowel & Education level - higher & \multicolumn{2}{|c|}{$\begin{array}{l}\text { Comorbidity; symptom type - pain, Age; sex; availability of vehicle } \\
\text { bleeding; first presenting at hospital; }\end{array}$} & Strong \\
\hline
\end{tabular}




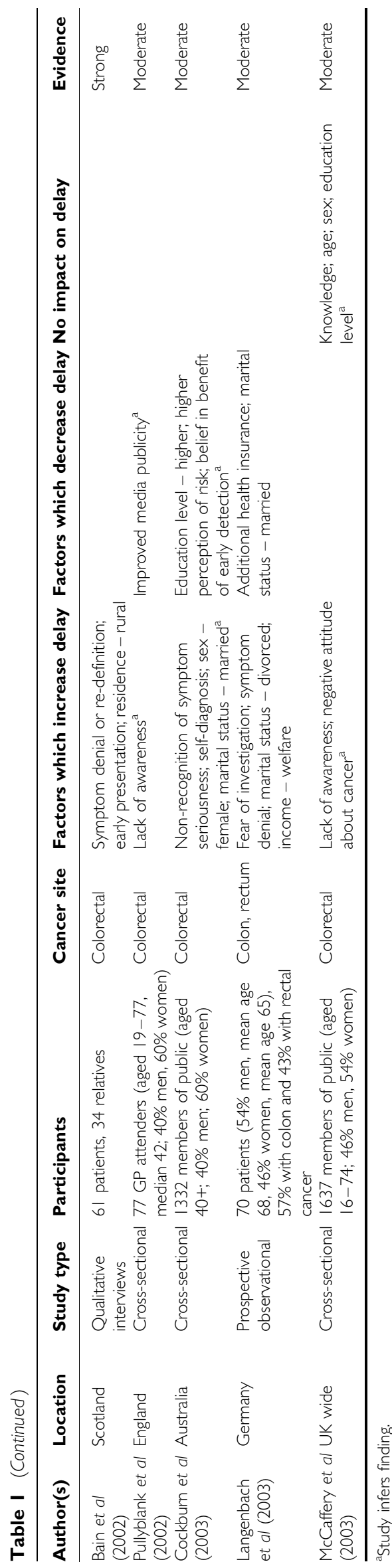

1999; Mariscal et al, 2001; McCaffery et al, 2003) and sex (Worden and Weisman, 1975; MacAdam, 1979; McDermott et al, 1981; Turunen and Peltokallio, 1982; Marshall and Funch, 1986; Robinson et al, 1986; Samet et al, 1988; Dent et al, 1990; Kemppainen et al, 1993; Porta et al, 1996; Mulcahy and O’Donoghue, 1997; Majumdar et al, 1999; Sladden et al, 1999; Young et al, 2000; Mariscal et al, 2001; Cockburn et al, 2003; McCaffery et al, 2003) had no impact on delay. Furthermore, there was no relationship between delay and lower socioeconomic status (Hackett et al, 1973; Worden and Weisman, 1975; MacAdam, 1979; MacArthur and Smith, 1984; Samet et al, 1988; Prohaska et al, 1990; Porta et al, 1996; Langenbach et al, 2003), although some studies suggested that this might be associated with increased delay. Rural residence (Robinson et al, 1986; Bain et al, 2002) and lower levels of education (Marshall and Funch, 1986; Dent et al, 1990; Vineis et al, 1993; Porta et al, 1996; Mariscal et al, 2001; Cockburn et al, 2003; McCaffery et al, 2003) were both found to increase delay.

\section{Factors influencing practitioner delay}

Twenty-nine papers considered factors that influence practitioner delay. More than three-quarters $(n=24)$ identified factors that increased delay and less than half $(n=12)$ factors that decreased delay (Figure 3).

\section{Practitioner behaviour}

The most commonly identified themes associated with delayed referral related to initial diagnosis and activity of the practitioner. Misdiagnosis, occurring either through treating patients symptomatically or attributing symptoms to a health problem other than colorectal cancer, resulted in increased time to referral (Spasov, 1978; Holliday and Hardcastle, 1979; Rubin et al, 1980; Zaichuk, 1980; Nilsson et al, 1982; Turunen and Peltokallio, 1982; Funch, 1985; Dixon et al, 1990; Mansson, 1990; Edwards et al, 1991; Harris and Simson, 1998; Roncoroni et al, 1999; Young et al, 2000). In addition, failure to examine the patient, usually rectal examination (Spasov, 1978; Holliday and Hardcastle, 1979; Rubin et al, 1980; Zaichuk, 1980; Turunen and Peltokallio, 1982; MacArthur and Smith, 1984; Dixon et al, 1990; Mansson, 1990; Tanum et al, 1991; Kemppainen et al, 1993; Roncoroni et al, 1999; Young et al, 2000; Langenbach et al, 2003), or receiving negative or false negative test results (Funch, 1985; Kemppainen et al, 1993; Harris and Simson, 1998) contributed to the delay. One qualitative study suggested that early presentation on the part of the patient could actually increase delay if disease went undetected or was misdiagnosed as benign (Bain et al, 2002). In addition, some patients identified practitioners as gatekeepers and a potential barrier to their referral since the patient waited for the GP to act on their behalf (Bain et al, 2002). Although limited, there is some evidence to suggest that appropriate referral and use of referral guidelines is associated with reduced delay (Holliday and Hardcastle, 1979; Debnath et al, 2002; Eccersley et al, 2003). Practitioners in rural areas were less likely to refer, due to the distance from specialist services (Sladden and Thomson, 1998) (Table 2).

\section{Presenting symptom}

Although the nature of symptoms will undoubtedly have contributed to referral decisions, it was difficult to reach definitive conclusions about their influence. For some patients, experiencing pain resulted in more rapid referral (Mariscal et al, 2001), whereas for others this had no impact (Young et al, 2000). Similarly, presenting with rectal bleeding could lead both to a more rapid (Sladden and Thomson, 1998; Mariscal et al, 2001) or more delayed outcome (Rubin et al, 1980; Mansson, 1990; Edwards et al, 


\begin{tabular}{l} 
Decreases \\
\hline Symptoms
\end{tabular}

Factor included only if supported by studies providing strong or moderate levels of evidence $\approx$ Found in single study; $*$ found in $\geqslant 50 \%$ of studies considering factor; $* *$ found in $\geqslant 75 \%$ of studies considering factor

Figure 3 Main factors associated with practitioner delay and direction of influence.

1991). Evaluation of the impact of tumour site on referral decision demonstrated that patients with rectal cancers were less likely to experience delay than those with colon cancers (MacAdam, 1979; Marshall and Funch, 1986; Robinson et al, 1986; Ratcliffe et al, 1989; Arbman et al, 1996).

\section{Patient history}

Consultation patterns related to obtaining a diagnosis were also found to be of relevance, with those patients, often women, who frequently consulted their general practitioner following a nonconclusive initial visit, more likely to experience delayed referral (MacAdam, 1979; Turunen and Peltokallio, 1982; Marshall and Funch, 1986). Similarly, those lacking continuity of care could also suffer delay, although a second opinion might precipitate referral (Bain et al, 2002). Patients with co-existing disease were likely to be referred more quickly (Mariscal et al, 2001).

\section{Patient characteristics}

There was some evidence relating to the impact of certain patient characteristics on practitioners' referral patterns. Older patients were in general referred more quickly (Nilsson et al, 1982; Turunen and Peltokallio, 1982; Pitluk and Poticha, 1983; Robinson et al, 1986; Sladden and Thomson, 1998), and there is some evidence of an association between delay and social class, with those from the lower end of the socioeconomic spectrum experiencing a longer wait to referral (MacArthur and Smith, 1984). There was however, no conclusive relationship between patient sex and referral delay (Turunen and Peltokallio, 1982; Marshall and Funch, 1986; Robinson et al, 1986; Arbman et al, 1996).

\section{DISCUSSION}

The importance of colorectal cancer in terms of its burden to society is well established; the benefits of presentation and diagnosis early in the course of the disease are clear. Yet although early diagnosis is desirable, it is also difficult and delays can occur at various points in the process. Understanding why delay occurs is the first step to reducing it. This paper presents a comprehensive, systematic review of the literature relating to the reasons for patient and primary care delay in the diagnosis of colorectal cancer. We found evidence of an association between delay and an extensive number of factors concentrated around four emergent themes: symptoms, patient history, patient characteristics and behaviour.

A strength of this review is the inclusion of studies in any language, reducing the potential for bias introduced by the exclusion of papers published in non-English language journals, which may be more inclined to show negative results (Egger et al, 1997). The main limitation of the review relates to substantial heterogeneity between included studies. The nature of the topic and variability in study quality and reporting made it neither possible nor appropriate to pool data for meta-analysis. Rather, we graded study evidence by the robustness of its methodology and analysis, allowing us to weight each study in our composite assessment of delay-related factors. A previous systematic review of delay in breast cancer concluded that most studies were of poor quality and that the strength of evidence was inadequate to inform development of strategies to shorten delay (Ramirez et al, 1999). Half of all studies included in this review provided strong evidence in relation to the factors they reported, and a further third provided moderate evidence. It is encouraging to note that most of these papers have been published since 1990; almost half in the last decade. Furthermore, we identified considerably more evidence than previous narrative and systematic reviews conducted as part of evidence-based guidelines (Carter and Winslet, 1998; Scottish Intercollegiate Guidelines Network, 2003).

One important finding of this review centres on the complex relationship between presentation behaviour and presenting symptoms. The evidence suggests that if delay is to be reduced, what is important is not simply patients' awareness of symptoms but rather their recognition and understanding of the potential seriousness of those symptoms. The implications of this are not 
Table 2 Practitioner associated delay factors

\begin{tabular}{|c|c|c|c|c|c|c|c|c|}
\hline Author(s) & Location & Study type & Participants & Cancer site & Factors which increase delay & Factors which decrease delay & No impact on delay & Evidence \\
\hline Spasov (1978) & Russia & $\begin{array}{l}\text { Retrospective } \\
\text { observational }\end{array}$ & 382 patients & Rectum & $\begin{array}{l}\text { Initial misdiagnosis; inadequate } \\
\text { investigation }\end{array}$ & & & $\begin{array}{l}\text { Unable to } \\
\text { determine }\end{array}$ \\
\hline $\begin{array}{l}\text { Holliday and } \\
\text { Hardcastle } \\
\text { (1979) }\end{array}$ & England & $\begin{array}{l}\text { Prospective } \\
\text { observational }\end{array}$ & $\begin{array}{l}200 \text { patients ( } 58 \% \text { men, mean age } \\
66 ; 42 \% \text { women mean age } 67 \text { ) }\end{array}$ & Colorectal & $\begin{array}{l}\text { Failure to examine; initial } \\
\text { misdiagnosis; inappropriate referral }\end{array}$ & & & Moderate \\
\hline $\begin{array}{l}\text { MacAdam } \\
\text { (1979) }\end{array}$ & England & $\begin{array}{l}\text { Prospective } \\
\text { observational }\end{array}$ & $\begin{array}{l}\text { I } 50 \text { patients ( } 79 \% \text { with colon/rectal } \\
\text { cancer), } 105 \mathrm{GPs}\end{array}$ & Colon, rectum & Cancer site - colon & Cancer site - rectum & Regular consulting rate of patient & Moderate \\
\hline $\begin{array}{l}\text { Rubin et al } \\
\text { 1980) }\end{array}$ & Israel & $\begin{array}{l}\text { Prospective } \\
\text { observational }\end{array}$ & $\begin{array}{l}100 \text { patients (aged } 36-85 \text {, mean } \\
64 ; 66 \% \text { men, } 34 \% \text { women) }\end{array}$ & Colorectal & $\begin{array}{l}\text { Initial misdiagnosis; failure to } \\
\text { examine; symptom type - bleeding }\end{array}$ & & & Strong \\
\hline Zaichuk (1980) & Russia & $\begin{array}{l}\text { Retrospective } \\
\text { observational }\end{array}$ & 55 patients & Rectum & $\begin{array}{l}\text { Failure to examine; initial } \\
\text { misdiagnosis }\end{array}$ & & & $\begin{array}{l}\text { Unable to } \\
\text { determine }\end{array}$ \\
\hline $\begin{array}{l}\text { Nilsson et al } \\
(1982)\end{array}$ & Sweden & $\begin{array}{l}\text { Retrospective } \\
\text { observational }\end{array}$ & $\begin{array}{l}284 \text { patients (aged } 20-99 ; 52 \% \\
\text { men, } 48 \% \text { women) }\end{array}$ & Colorectal & & $\begin{array}{l}\text { Adequate examination; Accurate } \\
\text { tests }^{\mathrm{a}}\end{array}$ & Patient age & Insufficient \\
\hline $\begin{array}{l}\text { Turunen and } \\
\text { Peltokallio } \\
\text { (1982) }\end{array}$ & Finland & $\begin{array}{l}\text { Prospective } \\
\text { observational }\end{array}$ & $\begin{array}{l}100 \text { patients ( } 45 \% \text { men, } 55 \% \\
\text { women }\end{array}$ & Colorectal & $\begin{array}{l}\text { Patient age }-<50 \text {; patient } \\
\text { sex }- \text { male; initial misdiagnosis; } \\
\text { failure to examine; frequent } \\
\text { attendance by patient }\end{array}$ & & & Moderate \\
\hline $\begin{array}{l}\text { Pitluk and } \\
\text { Poticha (1983) }\end{array}$ & Illinois, USA & $\begin{array}{l}\text { Retrospective } \\
\text { observational }\end{array}$ & $\begin{array}{l}826 \text { patients ( } 31 \text { aged } \leqslant 40 ; 45 \% \\
\text { men, } 55 \% \text { women) }\end{array}$ & Colorectal & Patient age - younger & & & Moderate \\
\hline $\begin{array}{l}\text { MacArthur and } \\
\text { Smith (1984) }\end{array}$ & England & $\begin{array}{l}\text { Prospective } \\
\text { observational }\end{array}$ & 127 patients & Colorectal & $\begin{array}{l}\text { Failure to examine; patient social } \\
\text { class - lower }\end{array}$ & $\begin{array}{l}\text { Symptom } \\
\text { type - constipation }\end{array}$ & & Strong \\
\hline Funch (1985) & $\begin{array}{l}\text { Washington, } \\
\text { USA }\end{array}$ & $\begin{array}{l}\text { Prospective } \\
\text { observational }\end{array}$ & $\begin{array}{l}294 \text { patients (aged } 18-85 ; 49 \% \\
\text { men, } 51 \% \text { women) }\end{array}$ & Colorectal & Initial misdiagnosis; inaccurate tests & & & Moderate \\
\hline $\begin{array}{l}\text { Marshall and } \\
\text { Funch (1986) }\end{array}$ & $\begin{array}{l}\text { Washington, } \\
\text { USA }\end{array}$ & $\begin{array}{l}\text { Prospective } \\
\text { observational }\end{array}$ & $\begin{array}{l}306 \text { patients (aged } 18-85 ; 50 \% \\
\text { men, } 50 \% \text { women) }\end{array}$ & Colorectal & $\begin{array}{l}\text { Patient sex - female; cancer } \\
\text { site - colon; frequent attendance by } \\
\text { patient }\end{array}$ & & & Strong \\
\hline $\begin{array}{l}\text { Robinson et al } \\
\text { (1986) }\end{array}$ & Israel & $\begin{array}{l}\text { Retrospective } \\
\text { observational }\end{array}$ & $\begin{array}{l}445 \text { patients ( } 54 \% \text { men, } 46 \% \\
\text { women) }\end{array}$ & Colorectal & Cancer site - rectum & & $\begin{array}{l}\text { Patient age; } \\
\text { patient sex }\end{array}$ & Strong \\
\hline $\begin{array}{l}\text { Ratcliffe et al } \\
\text { (1989) }\end{array}$ & England & $\begin{array}{l}\text { Prospective } \\
\text { observational }\end{array}$ & $\begin{array}{l}332 \text { patients (aged } 30-100 \text {, mean } \\
70 ; 51 \% \text { men, } 49 \% \text { women) }\end{array}$ & Colorectal & Cancer site - rectum & Cancer site - left sided carcinoma & & Strong \\
\hline $\begin{array}{l}\text { Dixon et al } \\
(1990)\end{array}$ & England & $\begin{array}{l}\text { Retrospective } \\
\text { observational }\end{array}$ & $\begin{array}{l}376 \text { patients (aged } 31-91 \text {, median } \\
67 \text { ) referred by }|5| \mathrm{GPs}\end{array}$ & Colorectal & $\begin{array}{l}\text { Initial misdiagnosis; failure to } \\
\text { examine }^{a}\end{array}$ & Awareness $^{\mathrm{a}}$ & & Insufficient \\
\hline $\begin{array}{l}\text { Mansson } \\
\text { (1990) }\end{array}$ & Sweden & $\begin{array}{l}\text { Retrospective } \\
\text { observational }\end{array}$ & $\begin{array}{l}42 \text { patients (aged } 45-92 ; 43 \% \\
\text { men, } 57 \% \text { women) }\end{array}$ & Colorectal & $\begin{array}{l}\text { Initial misdiagnosis; failure to } \\
\text { examine; symptom type - bleeding }\end{array}$ & & & Moderate \\
\hline $\begin{array}{l}\text { Edwards et al } \\
\text { (199I) }\end{array}$ & Wales & $\begin{array}{l}\text { Retrospective } \\
\text { observational }\end{array}$ & $\begin{array}{l}22 \text { patients (aged } 45-81 \text {, mean } 63 ; \\
50 \% \text { men, } 50 \% \text { women) }\end{array}$ & Anus & $\begin{array}{l}\text { Symptom type - bleeding; initial } \\
\text { misdiagnosis }\end{array}$ & & & Moderate \\
\hline $\begin{array}{l}\text { Tanum et al } \\
(1991)\end{array}$ & Norway & $\begin{array}{l}\text { Retrospective } \\
\text { observational }\end{array}$ & $\begin{array}{l}\text { I } 17 \text { patients (aged } 35-91 ; 21 \% \\
\text { men, } 79 \% \text { women) }\end{array}$ & Anus & Failure to examine ${ }^{a}$ & & & Insufficient \\
\hline $\begin{array}{l}\text { Jones and } \\
\text { Dudgeon } \\
\text { (1992) }\end{array}$ & England & $\begin{array}{l}\text { Retrospective } \\
\text { observational }\end{array}$ & $\begin{array}{l}245 \text { GPs, I } 465 \text { patients (> } 300 \\
\text { with colon cancer) }\end{array}$ & Colon & & Cancer site - colon & & Moderate \\
\hline $\begin{array}{l}\text { Kemppainen } \\
\text { et al (1993) }\end{array}$ & Finland & $\begin{array}{l}\text { Retrospective } \\
\text { observational }\end{array}$ & $\begin{array}{l}\text { I78 patients (aged } 27-97, \text { mean } \\
91 ; 44 \% \text { men, } 56 \% \text { women) }\end{array}$ & Colorectal & Failure to examine; inaccurate tests & & & Moderate \\
\hline $\begin{array}{l}\text { Arbman et al } \\
\text { (1996) }\end{array}$ & Sweden & $\begin{array}{l}\text { Retrospective } \\
\text { observational }\end{array}$ & $\begin{array}{l}554 \text { patients (aged } 30-95 ; 51 \% \\
\text { men, mean age } 70 ; 49 \% \text { women, } \\
\text { mean age } 72 \text { ), } 39 \% \text { with rectal and } \\
61 \% \text { with colon cancer }\end{array}$ & Colon, rectum & Patient sex - female & Cancer site - rectum & & Strong \\
\hline $\begin{array}{l}\text { Harris and } \\
\text { Simson (1998) }\end{array}$ & England & $\begin{array}{l}\text { Retrospective } \\
\text { observational }\end{array}$ & $\begin{array}{l}17 \text { patients (aged } 43-86 \text {, mean } 72 ; \\
59 \% \text { men, } 41 \% \text { women) }\end{array}$ & Colorectal & $\begin{array}{l}\text { Initial misdiagnosis; inaccurate } \\
\text { tests }\end{array}$ & & & Moderate \\
\hline $\begin{array}{l}\text { Sladden and } \\
\text { Thomson } \\
\text { (1998) }\end{array}$ & Australia & Cross-sectional & 68 GPs (aged 32-67; median 44) & Rectum & Practice location - rural & $\begin{array}{l}\text { Patient age - older; symptom type } \\
\text { - blood in toilet; no visible cause; } \\
\text { multiple episodes of bleeding }\end{array}$ & & Strong \\
\hline
\end{tabular}

\section{Clinical Studies}


without difficulty. For the majority of patients presenting to primary care, symptoms such as rectal bleeding and change in bowel habit are attributable to benign, self-limiting illness. Interpretation of these symptoms as benign will therefore usually be correct. However, for the minority of patients whose symptoms are due to colorectal cancer, delays if long enough may lead to more advanced stage disease and less chance of cure. Thus, considerable emphasis must be placed on highlighting the potentially significant nature of symptoms, despite their commonality. The challenge lies in achieving a suitable balance, which targets the appropriate population without creating undue fear, overburdening primary care services with patients seeking reassurance or clogging up scarce investigative services. This is particularly important given the paradoxical relationship that can exist between delay and fear of a potential cancer symptom.

Although some patients denied their symptoms or re-defined them in relation to benign disease, self-diagnosis and selftreatment were common themes across studies. This may go some way to explaining why patient delay was found to be greater for rectal than for colon cancers. It is likely that many people will associate rectal bleeding with haemorrhoids or some other benign ano-rectal problem. Embarrassment may deter presentation. As such, patients may not attend with the symptom until it becomes problematic; thus for example, the presence of pain reduces delay. Interestingly, patients with comorbidity also delayed less, perhaps due to their already frequent attendance in practice and the ease with which new problems could then be discussed.

The most common reasons for practitioner delay related to initial misdiagnosis and insufficient examination. This is in keeping with findings from previous reviews of delay in cancer diagnosis and is similar to those reported for hospital-related delay (Goodman and Irvin, 1993; Potter and Wilson, 1999). Lower gastrointestinal symptoms are common in patients presenting to primary care and the challenge of appropriate referral is a significant one. The complexity surrounding identification of those patients requiring further investigation has led to the production of several guidelines, all with the aim of facilitating earlier diagnosis. Implicit within these is examination, either to determine whether the patient has an abdominal or rectal mass, or to confirm the existence of a benign explanation. Yet, this review suggests that at least a quarter of patients and perhaps as many as three quarters do not receive a rectal examination (Holliday and Hardcastle, 1979; Rubin et al, 1980; Nilsson et al, 1982; Turunen and Peltokallio, 1982; MacArthur and Smith, 1984; Dixon et al, 1990; Kemppainen et al, 1993; Roncoroni et al, 1999; Young et al, 2000; Langenbach et al, 2003). The full impact of the use of referral guidelines is not yet clear; there is some evidence to suggest that they may reduce delay, but the strength of that evidence to date is limited. Furthermore, we found no intervention studies related to reducing patient or practitioner delay for colorectal cancer. Consequently, the impact of existing initiatives, such as guidelines, must be investigated further.

The NHS is currently rolling out a bowel cancer screening programme; it commenced in England and is due to achieve nationwide coverage by 2009 (http://cancerscreening.org.uk/bowel). The programme targets men and women aged 60-69 (50-74 in Scotland) and offers biennial screening via home faecal occult blood test kits. The full impact of the programme on patients' response to bowel symptoms is likely to be complex and will require evaluation. Nonetheless, it is likely that such screening will have some influence on pre-hospital delay, possibly through raising awareness of bowel symptoms and their potential seriousness, with consequent earlier presentation.

However, the bowel screening programme is aimed at detecting early stage disease in asymptomatic patients. Consequently, delays caused by some factors identified in this review, such as fear of cancer, denial of symptoms, initial practitioner misdiagnosis or failure to fully examine patients with rectal bleeding, will most 
likely be unaffected by the screening programme. Furthermore, a negative screening result may give patients false reassurance if they subsequently develop symptoms, an occurrence that has already been found to contribute to practitioner delay (Funch, 1985; Kemppainen et al, 1993; Harris and Simson, 1998).

The findings from this review would suggest that the way ahead, although clear, is also complex. If we are to reduce delay in the prehospital phase of colorectal cancer diagnosis, we must address two main areas. Firstly, we must overcome the dilemma faced by patients, that of when to categorise nonspecific symptoms as nonserious. Attributing symptoms to benign disease may be entirely appropriate and as such, difficult to influence. Achieving this may include public education, but it is also likely to involve greater awareness of how symptoms are interpreted in the context of preexisting disease, patient experience, social circumstances and life priorities. Such focus, away from ensuring recognition of symptoms and towards improving understanding of symptoms, will in turn require a shift in thinking on the part of the medical and research communities.

Secondly, we must influence the circumstances under which practitioners decide that further examination, investigation and referral are appropriate. This may require changes to existing guidelines, whereby physical examination and laboratory investigation are made explicit rather than implicit in the decision to refer. It may also require a change in practice, resulting in physical examination of all those who present with lower gastrointestinal symptoms, regardless of age, previous history or symptom duration. The initiation of such changes may already be underway, and indeed the recently published NICE Referral Guidelines for Suspected Cancer, which this review was intended to inform, now explicitly state that digital rectal examination should always be carried out in patients with unexplained lower GI symptoms (National Institute for Health and Clinical Excellence, 2005). What is certain is that pre-hospital delay in colorectal cancer is avoidable and it must be addressed if outcomes and survival are to be improved.

\section{ACKNOWLEDGEMENTS}

We acknowledge the support of Michere Beaumont, Catherine McNeill (Research Secretaries), Jane Goodfellow (Dissemination and Information Officer) and Madeline Young (Subject Librarian), University of Glasgow in carrying out this project. We thank David Mant for his input into the project, Joan Austoker for help in identifying international contacts, and Jolanta Astle, Simon Browne, Yulia Gaidakova, Karen Kane, Marina Novikova, Gaby Vojt and Phil Wilson for translation of non-English language articles. Finally, we thank the authors, cancer leads and other contacts who responded to our request for relevant work. This study was funded by the UK Department of Health Policy Research Programme (Project reference 1217522).

\section{REFERENCES}

Anon (1982) Cancer of the colon and rectum: public misconceptions and a gap in doctor-patient communications. Urban Health 11: 46-48

Anon (1986) Early detection of colorectal cancer. Cancer Nurs 9(6): 342

Arbman G, Nilsson E, Storgren-Fordell V, Sjodahl R (1996) A short diagnostic delay is more important for rectal cancer than for colonic cancer. Eur J Surg 162: 899-904

Australian Cancer Network (2006) Clinical practice guidelines for the prevention, early detection and management of colorectal cancer: a guide for general practitioners. www.cancerscreening.gov.au/internet/ screening/publishing.nsf/content/bw-gp-crc-guide/\$file/gp-crc-guide.pdf (accessed on 11 July 2007)

Bain NSC, Campbell NC, Ritchie LD, Cassidy J (2002) Striking the right balance in colorectal cancer - a qualitative study of rural and urban patients. Fam Pract 19(4): 369-374

Benson AB, Desch CE, Flynn PJ, Krause C, Loprinzi CL, Minsky BD, Petrelli NJ, Pfister DG, Smith TJ, Somerfield MR (2000) 2000 update of American Society of Clinical Oncology colorectal cancer surveillance guidelines. J Clin Oncol 18(20): 3586-3588

Byles JE, Redman S, Hennrikus D, Sanson-Fisher RW, Dickinson J (1992) Delay in consulting a medical practitioner about rectal bleeding. J Epidemiol Community Health 46: 241 - 244

Camilleri-Brennan J, Steele RJC (1999) A comparative study of knowledge and awareness of colorectal and breast cancer. Eur J Surg Oncol 25: $580-583$

Carter S, Winslet M (1998) Delay in the presentation of colorectal carcinoma: a review of causation. Int J Colorectal Dis 13: 27-31

Cockburn J, Paul C, Tzelepis F, McElduff P, Byles J (2003) Delay in seeking advice for symptoms that potentially indicate bowel cancer. Am J Health Behav 27(4): $401-407$

Curless R, French JM, Williams GV, James OFW (1994) Colorectal carcinoma: do elderly patients present differently? Age Ageing 23: $102-107$

de Nooijer J, Lechner L, de Vries H (2001) Help-seeking behaviour for cancer-symptoms: perceptions of patients and General Practitioners. Psychooncology 10(6): 469-478

Debnath D, Dielehner N, Gunning KA (2002) Guidelines, compliance, and effectiveness: a 12 months' audit in an acute district general healthcare trust on the two week rule for suspected colorectal cancer. Postgrad Med J 78: $748-751$
Dent OF, Goulston KJ, Tennant CC, Langeluddecke P, Mant A, Chapuis PH, Ward M, Bokey EL (1990) Rectal bleeding: patient delay in presentation. Dis Colon Rectum 33(10): $851-857$

Department of Health (2000) Referral Guidelines for Suspected Cancer. London: DoH

Devlin HB, Plant JA, Morris D (1973) The significance of the symptoms of carcinoma of the rectum. Surg Gyneacol Obstet 137: $399-402$

Dixon AR, Thornton-Holmes J, Cheetham NM (1990) General practitioners' awareness of colorectal cancer: a 10 year review. BMJ 301: $152-153$

Eccersley AJ, Wilson EM, Makris A, Novell JR (2003) Referral guidelines for colorectal cancer - do they work? Ann R Coll Surg Engl 85: 107-110

Edwards AT, Morus LC, Foster ME, Griffith GH (1991) Anal cancer: the case for earlier diagnosis. $J$ R Soc Med 84: 395-397

Egger M, Zellweger-Zahner T, Schneider M, Junker C, Lengeler C, Antes G (1997) Language bias in randomised controlled trials published in English and German. Lancet 350: 326-329

Funch DP (1985) Diagnostic delay in symptomatic colorectal cancer. Cancer 56: $2120-2124$

Galloway DJ, Burns HJG, Bear H, Jarret F, Boyle P, George WD (1984) Colorectal cancer in young adults. Clin Oncol 10: 205-211

Goodman D, Irvin TT (1993) Delay in the diagnosis and prognosis of carcinoma of the right colon. Br J Surg 80: 1327-1329

Hackett TP, Cassem NH, Raker JW (1973) Patient delay in cancer. N Engl J Med 289(1): 14-20

Harris GJC, Simson JNL (1998) Causes of late diagnosis in cases of colorectal cancer seen in a district general hospital over a 2-year period. Ann R Coll Surg Engl 80: 246-248

Holliday HW, Hardcastle JD (1979) Delay in diagnosis and treatment of symptomatic colorectal cancer. Lancet 1: 309-311

Jones RVH, Dudgeon TA (1992) Time between presentation and treatment of six common cancers: a study in Devon. Br J Gen Pract 42: $419-422$

Kemppainen M, Raiha I, Rajala T, Sourander L (1993) Delay in diagnosis of colorectal cancer in elderly patients. Age Ageing 22: 260-264

Langenbach MR, Schmidt J, Neumann J, Zirngibl H (2003) Delay in treatment of colorectal cancer: multifactorial problem. World J Surg 27: $304-308$ 
MacAdam DB (1979) A study in general practice of the symptoms and delay patterns in the diagnosis of gastrointestinal cancer. J R Coll Gen Pract 29: $723-729$

MacArthur C, Smith A (1984) Factors associated with speed of diagnosis, referral and treatment in colorectal cancer. J Epidemiol Community Health 38: $122-126$

MacDonald L, Freeling P (1986) Bowels: beliefs and behaviour. Fam Pract 3(2): $80-84$

Macdonald S, Macleod U, Campbell NC, Weller D, Mitchell E (2006) Systematic review of factors influencing patient and practitioner delay in diagnosis of upper gastrointestinal cancer. $\mathrm{Br} J$ Cancer 94: $1272-1280$

Majumdar SR, Fletcher RH, Evans AT (1999) How does colorectal cancer present? Symptoms, duration, and clues to location. Am J Gastroenterol 94(10): 3039-3045

Mansson J (1990) The diagnosis of colorectal cancer - experiences from the community of Kungsbacka, Sweden. Scand J Prim Health Care 8: 31 - 35

Marble K, Banerjee S, Greenwald L (1992) Colorectal carcinoma in young patients. J Surg Oncol 51: 179-182

Mariscal M, Llorca J, Prieto D, Delgado-Rodriguez M (2001) Determinants of the interval between the onset of symptoms and diagnosis in patients with digestive tract cancers. Cancer Detect Prevent 25(5): $420-429$

Marshall JR, Funch DP (1986) Gender and illness behavior among colorectal cancer patients. Women and Health 11(3-4): 67-82

McArdle CS, Hole DJ (2002) Outcome following surgery for colorectal cancer: analysis by hospital after adjustment for case-mix and deprivation. $\mathrm{Br}$ J Cancer 86: 331 - 335

McCaffery K, Wardle J, Waller J (2003) Knowledge, attitudes, and behavioural intentions in relation to the early detection of colorectal cancer in the United Kingdom. Prev Med 36: 525-535

McDermott F, Hughes E, Pihl E, Milne BJ, Price A (1981) Symptom duration and survival prospects in carcinoma of the rectum. Surg Gynecol Obstet 153(3): $321-326$

Mitchell E, Sullivan F (2001) A descriptive feast but an evaluative famine: systematic review of published articles on primary care computing during 1980-97. BMJ 322: 279-282

Mor V, Masterson-Allen S, Goldberg R, Guadagnoli E, Wool MS (1990) Prediagnostic symptom recognition and help-seeking among cancer patients. J Community Health 15(4): 253-266

Mulcahy HE, O'Donoghue DP (1997) Duration of colorectal cancer symptoms and survival: the effect of confounding clinical and pathological variables. Eur J Cancer 33(9): $1461-1467$

National Institute for Health and Clinical Excellence (2005) Referral Guidelines for Suspected Cancer: Clinical Guideline 27. London: NICE

Nichols S, Waters WE, Fraser JD, Wheeller MJ, Ingham SK (1981) Delay in the presentation of breast symptoms for consultant investigation. Community Med 3: 217-225

Nilsson E, Bolin S, Sjodahl R (1982) Carcinoma of the colon and rectum. Delay in diagnosis. Acta Chir Scand 148: 617-622

Parkin DM, Bray F, Ferlay J, Pisani P (2001) Estimating the world cancer burden: GLOBOCAN 2000. Int J Cancer 94: 153-156

Parkin DM, Pisani P, Ferlay J (1999) Estimates of the worldwide incidence of 25 major cancers in 1990. Int J Cancer 80: 827-841
Pitluk H, Poticha SM (1983) Carcinoma of the colon and rectum in patients less than 40 years of age. Surg Gynecol Obstet 157: $355-357$

Porta M, Gallen M, Belloc J, Malats N (1996) Predictors of the interval between onset of symptoms and first medical visit in patients with digestive tract cancer. Int J Oncol 8: $941-949$

Potter MA, Wilson RG (1999) Diagnostic delay in colorectal cancer. J R Coll Surg Edinb 44: 313-316

Prohaska TR, Funch D, Blesch KS (1990) Age patterns in symptom perception and illness behavior among colorectal cancer patients. Behav Health Aging 1(1): 27-39

Pullyblank AM, Dixon N, Dixon AR (2002) The impact of bowel cancer awareness week. Colorectal Dis 4: 483-485

Ramirez AJ, Westcombe AM, Burgess CC, Sutton S, Littlejohns P, Richards MA (1999) Factors predicting delayed presentation of symptomatic breast cancer: a systematic review. Lancet 353: $1127-1131$

Ratcliffe R, Kiff RS, Kingston RD, Walsh SH, Jeacock J (1989) Early diagnosis in colorectal cancer. Still no benefit? J R Coll Surg Edinb 34: $152-155$

Robinson E, Mohilever J, Zidan J, Sapir D (1986) Colorectal cancer: incidence, delay in diagnosis and stage of disease. Eur J Cancer Clin Oncol 22(2): $157-161$

Roncoroni L, Pietra N, Violi V, Sarli L, Choua O, Peracchia A (1999) Delay in the diagnosis and outcome of colorectal cancer: a prospective study. Eur J Surg Oncol 25: $173-178$

Rubin M, Zer M, Dintsman M (1980) Factors influencing delay in treatment of cancer of the rectum and colon in Israel. Isr J Med Sci 16(9-10): 641-645

Samet JM, Hunt WC, Lerchen ML, Goodwin JS (1988) Delay in seeking care for cancer symptoms: a population-based study of elderly New Mexicans. J Natl Cancer Inst 80(6): $432-438$

Scottish Intercollegiate Guidelines Network (2003) Management of Colorectal Cancer. A National Clinical Guideline 67. Edinburgh: Scottish Intercollegiate Guideline Network

Sladden MJ, Thomson AN (1998) How do general practitioners manage rectal bleeding? Aust Fam Physician 27(1-2): 78-82

Sladden MJ, Thomson AN, Lombard CJ (1999) Rectal bleeding in general practice patients. Aust Fam Physician 28(7): 750-754

Spasov GA (1978) Cause of late diagnosis in rectal cancer. Sov Med 2: $120-123$

Tanum G, Tveit K, Karlson KO (1991) Diagnosis of anal carcinoma doctor's finger still the best? Oncology 48: 383-386

Turunen MJ, Peltokallio P (1982) Delay in the diagnosis of colorectal cancer. Ann Chir Gynaecol 71: 277-282

Vineis P, Fornero G, Magnino A, Giacometti R, Ciccone G (1993) Diagnostic delay, clinical stage and social class: a hospital based study. J Epidemiol Community Health 47(3): 229-231

Worden JW, Weisman AD (1975) Psychosocial components of lagtime in cancer diagnosis. J Psychosom Res 19: 69-79

Young CJ, Sweeney JL, Hunter A (2000) Implications of delayed diagnosis in colorectal cancer. Aust N Z J Surg 70: 635-638

Zaichuk AI (1980) Reasons for the late diagnosis of rectal cancer. Vrach Delo 2: $66-67$ 